\title{
SLAVNOV AND GAUDIN-KOREPIN FORMULAS FOR MODELS WITHOUT $U(1)$ SYMMETRY: THE XXX CHAIN ON THE SEGMENT
}

\author{
S. BELLIARD AND R.A. PIMENTA
}

\begin{abstract}
We consider the isotropic spin- $\frac{1}{2}$ Heisenberg chain with the most general integrable boundaries. The scalar product between the on-shell Bethe vector and its off-shell dual, obtained by means of the modified algebraic Bethe ansatz, is given by a modified Slavnov formula. The corresponding Gaudin-Korepin formula, i.e., the square of the norm, is also obtained.
\end{abstract}

Introduction. The algebraic Bethe ansatz (ABA) 31, 30 is a powerful technique to study the spectral problem of quantum integrable models, as well as to construct their correlation functions and compute physical quantities [23. The possibility of expressing scalar products in a compact form [17, 18, 22, 32] is a crucial aspect of the method. Nevertheless, the application of the usual ABA to obtain the spectrum and the eigenvectors of quantum integrable models becomes problematic for some important cases, in particular in the presence of the non-diagonal boundaries. In the case of the Heisenberg spin chain on the segment, this is a consequence of the breaking of the $U(1)$ symmetry by off-diagonal boundaries. Many approaches have been developed to handle this problem, including generalizations of the Bethe ansatz to consider special non-diagonal boundaries, see for instance [9, 26, 4, 29, 1] and references therein, the SoV method [15, 14, 28, 13, 21, the functional method [16], the q-Onsager approach [8] and the non-polynomial solution from the homogeneous Baxter T-Q relation [25.

Recently, the ABA has been generalized to include models with general boundary couplings [3, 5, 11, 6, 2, . The modified algebraic Bethe ansatz (MABA) has a distinct feature: the creation operator used to construct the eigenstates has an off-shell structure which leads to an inhomogeneous term in the eigenvalues and in the Bethe equations of the model. We remember that such inhomogeneous term was firstly proposed in the context of the off-diagonal Bethe ansatz (ODBA) method (see [33] for a review) and recovered in the separation of variables (SoV) framework 24]. Let us also remark that the SoV basis [13] has been used to prove the off-shell equation for the creation operator in the MABA context 2 as well as to obtain the on-shell Bethe vector in the ODBA method [36, 37.

Once the spectral level is understood, the next natural step is to consider the evaluation of scalar products between Bethe vectors obtained in the MABA framework. The calculation of scalar products within this context is a primordial step to access the physical behavior of systems with general integrable open boundaries, since it paves the way to consider form factors and correlation functions. This task has been recently initiated in the case of the twisted XXX spin chain [7, a prototype model that can be described by the MABA. Modified Slavnov and Gaudin-Korepin formulas, i.e., compact expressions for the scalar product between an on-shell and an off-shell Bethe state and the square of the norm, have been conjectured. We propose in this note similar formulas for the XXX spin chain on the segment. They are given in terms of a determinant involving the inhomogeneous eigenvalue expression and a factor related to a certain expansion of the Bethe vector. Such new factor and the presence of an inhomogeneous eigenvalue motivate the nomenclature modified Slavnov and Gaudin-Korepin formulas for the models without $U(1)$ symmetry, to distinguish them from the diagonal or constrained cases known in the literature [34, 19, 20, 35]. 
Basic formalism. Let us recall the fundamental objects of quantum inverse scattering method for models on the segment [30]. The main object is the R-matrix, solution of the Yang-Baxter equation,

$$
R_{a b}(u-v) R_{a c}(u-w) R_{b c}(v-w)=R_{b c}(v-w) R_{a c}(u-w) R_{a b}(u-v),
$$

defined in the tensor product of complex vector spaces $V_{a} \otimes V_{b} \otimes V_{c}$ with $V_{i}=\mathbb{C}^{2}$. In the case of the XXX spin chain, the associated $R$-matrix is called the rational $R$-matrix and it is given by,

$$
R(u)=u+P,
$$

where $P=\sigma^{+} \otimes \sigma^{-}+\sigma^{-} \otimes \sigma^{+}+\frac{1}{2}\left(1+\sigma^{z} \otimes \sigma^{z}\right)$ is the permutation operator!. This $R$-matrix is $G L(2)$ invariant,

$$
[R(u), \mathscr{Q} \otimes \mathscr{Q}]=0,
$$

for any $\mathscr{Q}$ in $G L(2)$. For quantum integrable models on the segment additional objects must be considered, namely, the K-matrix, solution of the reflection equation,

$$
R_{a b}(u-v) K_{a}^{-}(u) R_{a b}(u+v) K_{b}^{-}(v)=K_{b}^{-}(v) R_{a b}(u+v) K_{a}^{-}(u) R_{a b}(u-v),
$$

and the dual K-matrix, solution of the dual reflection equation,

$$
\begin{aligned}
& R_{a b}(-u+v)\left(K_{a}^{+}(u)\right)^{t_{a}} R_{a b}(-u-v-2)\left(K_{b}^{+}(v)\right)^{t_{b}} \\
& \quad=\left(K_{b}^{+}(v)\right)^{t_{b}} R_{a b}(-u-v-2)\left(K_{a}^{+}(u)\right)^{t_{a}} R_{a b}(-u+v),
\end{aligned}
$$

where $t_{i}$ denotes the transposition in the $i$ th space. The most general scalar solutions of the equations (4) and (5) are given by [12],

$$
K^{+}(u)=\left(\begin{array}{cc}
q+u+1 & \xi^{+}(u+1) \\
\xi^{-}(u+1) & q-u-1
\end{array}\right), \quad K^{-}(u)=\left(\begin{array}{cc}
p+u & \eta^{+} u \\
\eta^{-} u & p-u
\end{array}\right),
$$

where $\left\{q, \xi^{+}, \xi^{-}, p, \eta^{+}, \eta^{-}\right\} \in \mathbb{C}^{6}$ are generic parameters. Due to the $G L(2)$ invariance, we can choose $\eta^{ \pm}=0$, which we will consider in the remaining of the text, without losing generality. The R-matrix and the K-matrices allow us to construct the following transfer matrix ,

$$
t(u)=\operatorname{Tr}_{a}\left(K_{a}^{+}(u) K_{a}(u)\right), \quad K_{a}(u)=T_{a}(u) K_{a}^{-}(u) \hat{T}_{a}(u)=\left(\begin{array}{cc}
\mathscr{A}(u) & \mathscr{B}(u) \\
\mathscr{C}(u) & \mathscr{D}(u)+\frac{1}{2 u+1} \mathscr{A}(u)
\end{array}\right)_{a},
$$

where the double-row monodromy matrix $K_{a}(u)$ is built by Sklyanin's dressing procedure for the $K^{-}$matrix, and the bulk monodromy matrices are given by,

$$
T_{a}(u)=R_{a 1}\left(u-\theta_{1}\right) \cdots R_{a N}\left(u-\theta_{N}\right), \quad \hat{T}_{a}(u)=R_{a N}\left(u+\theta_{N}\right) \cdots R_{a 1}\left(u+\theta_{1}\right),
$$

with the free parameters $\left\{\theta_{1}, \ldots \theta_{N}\right\}$ called inhomogeneity parameters. The operator entries of $K_{a}(u)$ act on the quantum space $\mathcal{H}=\otimes_{i=1}^{N} \mathbb{C}^{2}$ and satisfy commutation relations given in appendix $\mathrm{A}$. In terms of these operators, the transfer matrix reads,

$$
t(u)=\alpha(u) \mathscr{A}(u)+\delta(u) \mathscr{D}(u)+\beta(u) \mathscr{B}(u)+\gamma(u) \mathscr{C}(u),
$$

where

$$
\alpha(u)=\phi(u)(q+u), \quad \delta(u)=q-(u+1), \quad \beta(u)=\xi^{-}(u+1), \quad \gamma(u)=\xi^{+}(u+1),
$$

with

$$
\phi(u)=\frac{2(u+1)}{2 u+1}
$$

$$
{ }_{1}^{1} \sigma^{z}=\left(\begin{array}{cc}
1 & 0 \\
0 & -1
\end{array}\right), \quad \sigma^{+}=\left(\begin{array}{ll}
0 & 1 \\
0 & 0
\end{array}\right), \quad \sigma^{-}=\left(\begin{array}{ll}
0 & 0 \\
1 & 0
\end{array}\right), \quad \sigma^{x}=\sigma^{+}+\sigma^{-}, \quad \sigma^{y}=i\left(\sigma^{-}-\sigma^{+}\right) .
$$


Two transfer matrices at different values of their spectral parameter commute, i.e., $[t(u), t(v)]=0$. Therefore, the expansion of the transfer matrix $t(u)$ with respect to the spectral parameter $u$ provides a set of commuting operators, among which the isotropic Heisenberg Hamiltonian, given by,

$$
H=\frac{1}{q}\left(\sigma_{1}^{z}+\xi^{+} \sigma_{1}^{+}+\xi^{-} \sigma_{1}^{-}\right)+\sum_{n=1}^{N-1}\left(\sigma_{n}^{x} \otimes \sigma_{n+1}^{x}+\sigma_{n}^{y} \otimes \sigma_{n+1}^{y}+\sigma_{n}^{z} \otimes \sigma_{n+1}^{z}\right)+\frac{1}{p} \sigma_{N}^{z}
$$

The main problem is thus to find the eigenvectors and eigenvalues of the transfer matrix. Finally, let us recall that, due to the diagonal form of the $K^{-}$matrix, there exist a highest weight vector and its dual, namely,

$$
|\Omega\rangle=\otimes_{i=1}^{N}\left(\begin{array}{l}
1 \\
0
\end{array}\right), \quad\langle\Omega|=\otimes_{i=1}^{N}\left(\begin{array}{ll}
1 & 0
\end{array}\right),
$$

such that,

$$
\begin{aligned}
& \mathscr{A}(u)|\Omega\rangle=\Lambda_{1}(u)|\Omega\rangle, \quad \mathscr{D}(u)|\Omega\rangle=\Lambda_{2}(u)|\Omega\rangle, \quad \mathscr{C}(u)|\Omega\rangle=0, \\
& \langle\Omega| \mathscr{A}(u)=\langle\Omega| \Lambda_{1}(u), \quad\langle\Omega| \mathscr{D}(u)=\langle\Omega| \Lambda_{2}(u), \quad\langle\Omega| \mathscr{B}(u)=0,
\end{aligned}
$$

where

$$
\Lambda_{1}(u)=(u+p) \prod_{i=1}^{N}\left(u+1-\theta_{i}\right)\left(u+1+\theta_{i}\right), \quad \Lambda_{2}(u)=\phi(-u-1)(p-u-1) \prod_{i=1}^{N}\left(u-\theta_{i}\right)\left(u+\theta_{i}\right) .
$$

Modified algebraic Bethe ansatz. The Bethe vector for the present model was firstly conjectured in $\underline{3}$, and we review its obtainment here. The key point is to introduce a similarity transformation with a two-fold aim: to bring the original transfer matrix expression (9) to a modified diagonal form and to define a modified creation operator. Indeed, let us introduce,

$$
\mathscr{Q}=\left(\begin{array}{cc}
\xi^{+} & \rho \\
-\rho & \xi^{-}
\end{array}\right), \quad \rho=1-\sqrt{1+\xi^{+} \xi^{-}}
$$

which diagonalizes the $K^{+}$matrix,

$$
\bar{K}_{a}^{+}(u)=\mathscr{Q}_{a}^{-1} K_{a}^{+}(u) \mathscr{Q}_{a}=\left(\begin{array}{cc}
q+(1+u)(1-\rho) & 0 \\
0 & q-(1+u)(1-\rho)
\end{array}\right)_{a},
$$

and, as a consequence, the transfer matrix (7) can be written as,

$$
t(u)=\operatorname{Tr}_{a}\left(\bar{K}_{a}^{+}(u) \bar{K}_{a}(u)\right), \quad \bar{K}_{a}(u)=\mathscr{Q}_{a}^{-1} K_{a}^{-}(u) \mathscr{Q}_{a}=\left(\begin{array}{cc}
\overline{\mathscr{A}}(u) & \overline{\mathscr{B}}(u) \\
\overline{\mathscr{C}}(u) & \overline{\mathscr{D}}(u)+\frac{1}{2 u+1} \overline{\mathscr{A}}(u)
\end{array}\right)_{a} .
$$

The modified double-row monodromy operators are given explicitly by,

$$
\begin{aligned}
& \overline{\mathscr{A}}(u)=\frac{1}{2(\rho-1)}\left((\rho \phi(u)-2) \mathscr{A}(u)+\rho \mathscr{D}(u)-\xi^{-} \mathscr{B}(u)-\xi^{+} \mathscr{C}(u)\right), \\
& \overline{\mathscr{D}}(u)=\frac{1}{2(\rho-1)}\left((\rho \phi(-u-1)-2) \mathscr{D}(u)+\rho \phi(u) \phi(-u-1) \mathscr{A}(u)+\xi^{-} \phi(u) \mathscr{B}(u)+\xi^{+} \phi(u) \mathscr{C}(u)\right), \\
& \overline{\mathscr{B}}(u)=\frac{1}{2(\rho-1)}\left(\xi^{-} \phi(-u-1) \mathscr{A}(u)-\xi^{-} \mathscr{D}(u)+\frac{\xi^{-2}}{\rho} \mathscr{B}(u)-\rho \mathscr{C}(u)\right), \\
& \overline{\mathscr{C}}(u)=\frac{1}{2(\rho-1)}\left(\xi^{+} \phi(-u-1) \mathscr{A}(u)-\xi^{+} \mathscr{D}(u)-\rho \mathscr{B}(u)+\frac{\xi^{+2}}{\rho} \mathscr{C}(u)\right) .
\end{aligned}
$$

Since $\bar{K}_{a}^{+}(u)$ is a diagonal matrix, the transfer matrix acquires a modified diagonal form, namely,

$$
t(u)=\bar{\alpha}(u) \overline{\mathscr{A}}(u)+\bar{\delta}(u) \overline{\mathscr{D}}(u),
$$


with

$$
\bar{\alpha}(u)=\phi(u)(q+u(1-\rho)), \quad \bar{\delta}(u)=q-(1+u)(1-\rho) .
$$

As a consequence of (3), the operators $\{\overline{\mathscr{A}}(u), \overline{\mathscr{B}}(u), \overline{\mathscr{C}}(u), \overline{\mathscr{D}}(u)\}$ satisfy the same commutation relations as $\{\mathscr{A}(u), \mathscr{B}(u), \mathscr{C}(u), \mathscr{D}(u)\}$, see appendix 太. This means that the Bethe state can be created by the action of the operator $(\overline{\mathscr{C}}(u)) \overline{\mathscr{B}}(u)$ on the (dual) highest vector (13). Indeed, let us defind,

$$
\left|\Psi^{M}(\bar{u})\right\rangle=\overline{\mathscr{B}}\left(u_{1}\right) \ldots \overline{\mathscr{B}}\left(u_{M}\right)|\Omega\rangle=\overline{\mathscr{B}}(\bar{u})|\Omega\rangle
$$

and

$$
\left\langle\Psi^{M}(\bar{u})\right|=\langle\Omega| \overline{\mathscr{C}}\left(u_{1}\right) \ldots \overline{\mathscr{C}}\left(u_{M}\right)=\langle\Omega| \overline{\mathscr{C}}(\bar{u}) .
$$

The repeated use of the relations (6163165) alows us to obtain,

$$
\begin{aligned}
& \overline{\mathscr{A}}(u) \overline{\mathscr{B}}(\bar{u})=f(u, \bar{u}) \overline{\mathscr{B}}(\bar{u}) \overline{\mathscr{A}}(u) \\
& \quad+\sum_{i=1}^{M} g\left(u, u_{i}\right) f\left(u_{i}, \bar{u}_{i}\right) \overline{\mathscr{B}}\left(\left\{u, \bar{u}_{i}\right\}\right) \overline{\mathscr{A}}\left(u_{i}\right)+w\left(u, u_{i}\right) h\left(u_{i}, \bar{u}_{i}\right) \overline{\mathscr{B}}\left(\left\{u, \bar{u}_{i}\right\}\right) \overline{\mathscr{D}}\left(u_{i}\right), \\
& \overline{\mathscr{D}}(u) \overline{\mathscr{B}}(\bar{u})=h(u, \bar{u}) \overline{\mathscr{B}}(\bar{u}) \overline{\mathscr{D}}(u) \\
& \quad+\sum_{i=1}^{M} k\left(u, u_{i}\right) h\left(u_{i}, \bar{u}_{i}\right) \overline{\mathscr{B}}\left(\left\{u, \bar{u}_{i}\right\}\right) \overline{\mathscr{D}}\left(u_{i}\right)+n\left(u, u_{i}\right) f\left(u_{i}, \bar{u}_{i}\right) \overline{\mathscr{B}}\left(\left\{u, \bar{u}_{i}\right\}\right) \overline{\mathscr{A}}\left(u_{i}\right),
\end{aligned}
$$

while the use of (62164]66) gives us,

$$
\begin{aligned}
& \overline{\mathscr{C}}(\bar{u}) \overline{\mathscr{A}}(u)=f(u, \bar{u}) \overline{\mathscr{A}}(u) \overline{\mathscr{C}}(\bar{u}) \\
& \quad+\sum_{i=1}^{M} g\left(u, u_{i}\right) f\left(u_{i}, \bar{u}_{i}\right) \overline{\mathscr{A}}\left(u_{i}\right) \overline{\mathscr{C}}\left(\left\{u, \bar{u}_{i}\right\}\right)+w\left(u, u_{i}\right) h\left(u_{i}, \bar{u}_{i}\right) \overline{\mathscr{D}}\left(u_{i}\right) \overline{\mathscr{C}}\left(\left\{u, \bar{u}_{i}\right\}\right), \\
& \overline{\mathscr{C}}(\bar{u}) \overline{\mathscr{D}}(u)=h(u, \bar{u}) \overline{\mathscr{D}}(u) \overline{\mathscr{C}}(\bar{u}) \\
& \quad+\sum_{i=1}^{M} k\left(u, u_{i}\right) h\left(u_{i}, \bar{u}_{i}\right) \overline{\mathscr{D}}\left(u_{i}\right) \overline{\mathscr{C}}\left(\left\{u, \bar{u}_{i}\right\}\right)+n\left(u, u_{i}\right) f\left(u_{i}, \bar{u}_{i}\right) \overline{\mathscr{A}}\left(u_{i}\right) \overline{\mathscr{C}}\left(\left\{u, \bar{u}_{i}\right\}\right) .
\end{aligned}
$$

In order to evaluate the action of the transfer matrix on (23) and (24), in addition to (25) and (26), we also need to compute the action of the operators $\overline{\mathscr{A}}(u)$ and $\overline{\mathscr{D}}(u)$ on (13). It is a modified action, namely,

$$
\begin{aligned}
& \overline{\mathscr{A}}(u)|\Omega\rangle=\Lambda_{1}(u)|\Omega\rangle-\frac{\rho}{\xi^{-}} \overline{\mathscr{B}}(u)|\Omega\rangle, \quad \overline{\mathscr{D}}(u)|\Omega\rangle=\Lambda_{2}(u)|\Omega\rangle+\frac{\rho}{\xi^{-}} \phi(u) \overline{\mathscr{B}}(u)|\Omega\rangle, \\
& \overline{\mathscr{C}}(u)|\Omega\rangle=\frac{\rho}{\xi^{-}}\left(\phi(-u-1) \Lambda_{1}(u)-\Lambda_{2}(u)\right)|\Omega\rangle-\left(\frac{\rho}{\xi^{-}}\right)^{2} \overline{\mathscr{B}}(u)|\Omega\rangle, \\
& \langle\Omega| \overline{\mathscr{A}}(u)=\Lambda_{1}(u)\langle\Omega|-\frac{\rho}{\xi^{+}}\langle\Omega| \overline{\mathscr{C}}(u), \quad\langle\Omega| \overline{\mathscr{D}}(u)=\Lambda_{2}(u)\langle\Omega|+\frac{\rho}{\xi^{+}} \phi(u)\langle\Omega| \overline{\mathscr{C}}(u), \\
& \langle\Omega| \overline{\mathscr{B}}(u)=\frac{\rho}{\xi^{+}}\left(\phi(-u-1) \Lambda_{1}(u)-\Lambda_{2}(u)\right)\langle\Omega|-\left(\frac{\rho}{\xi^{+}}\right)^{2}\langle\Omega| \overline{\mathscr{C}}(u) .
\end{aligned}
$$

\footnotetext{
${ }^{2}$ Hereafter, we will use the following compact notation. A set of $M$ variables $\left\{u_{1}, u_{2}, \ldots, u_{M}\right\}$ is denoted by $\bar{u}$ with \# $\bar{u}=M$. If the $i$ th element is removed, we indicate $\bar{u}_{i}=\bar{u} / u_{i}$ and, if we also remove the element $u_{j}$, we denote $\bar{u}_{i j}=\bar{u} /\left\{u_{i}, u_{j}\right\}$. For products of functions $($ e.g. $f(u, v))$ or of operators $\mathscr{O}($ e.g. $\overline{\mathscr{B}})$, we use the convention

$$
f(u, \bar{u})=\prod_{i=1}^{M} f\left(u, u_{i}\right), \quad f(\bar{v}, \bar{u})=\prod_{i=1}^{M} \prod_{j=1}^{M} f\left(v_{j}, u_{i}\right), \quad f\left(u_{i}, \bar{u}_{i}\right)=\prod_{j=1, j \neq i}^{M} f\left(u_{i}, u_{j}\right), \quad \mathscr{O}(\bar{u})=\prod_{k=1}^{M} \mathscr{O}\left(u_{k}\right) .
$$
}


Using the multiple actions (25]26) together with (27/28), we obtain,

$$
\begin{aligned}
& t(u)\left|\Psi^{M}(\bar{u})\right\rangle=\Lambda_{d}^{M}(u, \bar{u})\left|\Psi^{M}(\bar{u})\right\rangle+\sum_{i=1}^{M} F\left(u, u_{i}\right) E_{d}^{M}\left(u_{i}, \bar{u}_{i}\right)\left|\Psi^{M}\left(\left\{u, \bar{u}_{i}\right\}\right)\right\rangle \\
& +\frac{\rho(\rho-1)}{\xi^{-}} 2(u+1) \overline{\mathscr{B}}(u)\left|\Psi^{M}(\bar{u})\right\rangle, \\
& \left\langle\Psi^{M}(\bar{u})\right| t(u)=\left\langle\Psi^{M}(\bar{u})\right| \Lambda_{d}^{M}(u, \bar{u})+\sum_{i=1}^{M} F\left(u, u_{i}\right) E_{d}^{M}\left(u_{i}, \bar{u}_{i}\right)\left\langle\Psi^{M}\left(\left\{u, \bar{u}_{i}\right\}\right)\right| \\
& +\frac{\rho(\rho-1)}{\xi^{+}} 2(u+1)\left\langle\Psi^{M}(\bar{u})\right| \overline{\mathscr{C}}(u),
\end{aligned}
$$

where

$$
\begin{aligned}
& \Lambda_{d}^{M}(u, \bar{u})=\bar{\alpha}(u) \Lambda_{1}(u) f(u, \bar{u})+\bar{\delta}(u) \Lambda_{2}(u) h(u, \bar{u}), \\
& E_{d}^{M}\left(u_{i}, \bar{u}_{i}\right)=-\phi\left(-u_{i}-1\right) \bar{\alpha}\left(u_{i}\right) \Lambda_{1}\left(u_{i}\right) f\left(u_{i}, \bar{u}_{i}\right)+\phi\left(u_{i}\right) \bar{\delta}\left(u_{i}\right) \Lambda_{2}\left(u_{i}\right) h\left(u_{i}, \bar{u}_{i}\right),
\end{aligned}
$$

with

$$
F(u, v)=-\frac{\phi(u)(2 u+1)}{\phi(v) Q(u, v)}, \quad Q(u, v)=(u-v)(u+v+1) .
$$

The new terms in (29|30), i.e., the proportional terms to $\overline{\mathscr{B}}(u)\left|\Psi^{M}(\bar{u})\right\rangle$ and $\left\langle\Psi^{M}(\bar{u})\right| \overline{\mathscr{C}}(u)$, are characteristic of MABA approach. If the number of creation operators equals the length of the chain, i.e., $M=N$, these terms are given by,

$$
\begin{aligned}
& \frac{\rho(\rho-1)}{\xi^{-}} 2(u+1) \overline{\mathscr{B}}(u)\left|\Psi^{N}(\bar{u})\right\rangle=\Lambda_{g}^{N}(u, \bar{u})\left|\Psi^{N}(\bar{u})\right\rangle+\sum_{i=1}^{N} F\left(u, u_{i}\right) E_{g}^{N}\left(u_{i}, \bar{u}_{i}\right)\left|\Psi^{N}\left(\left\{u, \bar{u}_{i}\right\}\right)\right\rangle, \\
& \frac{\rho(\rho-1)}{\xi^{+}} 2(u+1)\left\langle\Psi^{N}(\bar{u})\right| \overline{\mathscr{C}}(u)=\left\langle\Psi^{N}(\bar{u})\right| \Lambda_{g}^{N}(u, \bar{u})+\sum_{i=1}^{N} F\left(u, u_{i}\right) E_{g}^{N}\left(u_{i}, \bar{u}_{i}\right)\left\langle\Psi^{N}\left(\left\{u, \bar{u}_{i}\right\}\right)\right|,
\end{aligned}
$$

with

$$
\Lambda_{g}^{N}(u, \bar{u})=\rho \tilde{\phi}(u) \frac{\Lambda_{1}(u) \Lambda_{2}(u)}{Q(u, \bar{u})}, \quad E_{g}^{N}\left(u_{i}, \bar{u}_{i}\right)=\rho \frac{\tilde{\phi}\left(u_{i}\right)}{2 u_{i}+1} \frac{\Lambda_{1}\left(u_{i}\right) \Lambda_{2}\left(u_{i}\right)}{Q\left(u_{i}, \bar{u}_{i}\right)}, \quad \tilde{\phi}(u)=\frac{(u+1)(2 u+1)}{(p+u)(p-u-1)},
$$

and $\# \bar{u}=N$. The equations (33|34) are the central relations in the MABA. Their proof follows from the rational limit of the proof given in 2 for the XXZ case. We arrive, combining (29) and (33), to the final off-shell equation satisfied by the left and right Bethe vectors,

$$
\begin{aligned}
& t(u)\left|\Psi^{N}(\bar{u})\right\rangle=\Lambda^{N}(u, \bar{u})\left|\Psi^{N}(\bar{u})\right\rangle+\sum_{i=1}^{N} F\left(u, u_{i}\right) E^{N}\left(u_{i}, \bar{u}_{i}\right)\left|\Psi^{N}\left(\left\{u, \bar{u}_{i}\right\}\right)\right\rangle, \\
& \left\langle\Psi^{N}(\bar{u})\right| t(u)=\left\langle\Psi^{N}(\bar{u})\right| \Lambda^{N}(u, \bar{u})+\sum_{i=1}^{N} F\left(u, u_{i}\right) E^{N}\left(u_{i}, \bar{u}_{i}\right)\left\langle\Psi^{N}\left(\left\{u, \bar{u}_{i}\right\}\right)\right|,
\end{aligned}
$$

where the eigenvalue and the corresponding Bethe equations are given by,

$$
\Lambda^{N}(u, \bar{u})=\Lambda_{d}^{N}(u, \bar{u})+\Lambda_{g}^{N}(u, \bar{u}), \quad E^{N}\left(u_{i}, \bar{u}_{i}\right)=E_{d}^{N}\left(u_{i}, \bar{u}_{i}\right)+E_{g}^{N}\left(u_{i}, \bar{u}_{i}\right)
$$

with $\# u=N$. We recall here that the eigenvalue expression (38) was firstly obtained in [10, and it was further developed in 27. 
Modified Slavnov and Gaudin-Korepin formulas. We are now in position to investigate the scalar product between the Bethe vectors and its dual, namely,

$$
S^{N}(\bar{u} \mid \bar{v})=\left\langle\Psi^{N}(\bar{u}) \mid \Psi^{N}(\bar{v})\right\rangle .
$$

Considering $\left\langle\Psi^{N}(\bar{u})\right|$ on-shell, i.e., $E^{N}\left(u_{i}, \bar{u}_{i}\right)=0$ for $i=1, \ldots N$, we conjecture the following modified Slavnov formula,

$$
\hat{S}^{N}(\bar{u}, \bar{v})=\left(\frac{\rho-2}{2(\rho-1)^{2}}\right)^{N} W_{0}^{N}(\bar{u}) \frac{\operatorname{Det}_{N}\left(\frac{\partial}{\partial u_{i}} \Lambda^{N}\left(v_{j}, \bar{u}\right)\right)}{\operatorname{Det}_{N}\left(V\left(v_{i}, u_{j}\right)\right)}
$$

where

$$
W_{0}^{N}(\bar{u})=\left(\frac{2(\rho-1)}{\xi^{-}}\right)^{N}\langle 0|\overline{\mathscr{B}}(\bar{u})| 0\rangle, \quad V\left(v_{i}, u_{j}\right)=\frac{\left(2 u_{j}+1\right) 2\left(v_{i}+1\right)}{Q\left(v_{i}, u_{j}\right)}
$$

with $\# \bar{u}=N$. The factor $W_{0}^{N}(\bar{u})$ is related to the expansion of the Bethe vector in the basis of the original operators $\{\mathscr{A}(u), \mathscr{B}(u), \mathscr{C}(u), \mathscr{D}(u)\}$ and it is given in appendix B, If, on the other hand, $\left|\Psi^{N}(\bar{v})\right\rangle$ is on-shell, namely $E^{N}\left(v_{i}, \bar{v}_{i}\right)=0$ for $i=1, \ldots N$, the respective modified formula is obtained by interchanging $u_{i} \leftrightarrow v_{i}$ in the right-hand side of (40),

$$
\tilde{S}(\bar{u}, \bar{v})=\left(\frac{\rho-2}{2(\rho-1)^{2}}\right)^{N} W_{0}^{N}(\bar{v}) \frac{\operatorname{Det}_{N}\left(\frac{\partial}{\partial v_{i}} \Lambda^{N}\left(u_{j}, \bar{v}\right)\right)}{\operatorname{Det}_{N}\left(V\left(u_{i}, v_{j}\right)\right)} .
$$

The modified Gaudin-Korepin formula can be obtained by taking the limit $\bar{v}=\bar{u}$ in (40). In order to do that we firstly rewrite the Cauchy-like determinant as,

$$
\operatorname{Det}_{N}\left(V\left(v_{i}, u_{j}\right)\right)=\frac{\prod_{i=1}^{N}\left(2 u_{i}+1\right) 2\left(v_{i}+1\right) \prod_{i<j}^{N} Q\left(u_{j}, u_{i}\right) Q\left(v_{i}, v_{j}\right)}{\prod_{i, j=1}^{N} Q\left(v_{j}, u_{i}\right)},
$$

and, next, place the term $\prod_{i, j=1}^{N} Q\left(v_{j}, u_{i}\right)$ along with the Jacobian. We can then perform the limit,

$$
\lim _{v_{j} \rightarrow u_{j}}\left(\prod_{k=1}^{N} Q\left(v_{j}, u_{k}\right) \frac{\partial}{\partial u_{i}} \Lambda^{N}\left(v_{j}, \bar{u}\right)\right)
$$

using l'Hospital's rule. As a result we obtain the following expression for the square of the norm,

$$
\mathscr{N}^{N}(\bar{u})=\left(\frac{\rho-2}{2(\rho-1)^{2}}\right)^{N} W_{0}(\bar{u}) \frac{\operatorname{det}_{N}\left(G_{i j}\right)}{\prod_{i=1}^{N} 2\left(u_{i}+1\right) \prod_{i<j}^{N} Q\left(u_{j}, u_{i}\right) Q\left(u_{i}, u_{j}\right)},
$$

where the matrix elements $G_{i j}$ for $i, j=1, \ldots N$ are given by,

$$
\begin{aligned}
G_{i i} & =Q\left(u_{i}, \bar{u}_{i}\right) \frac{\partial E^{N}\left(u_{i}, \bar{u}_{i}\right)}{\partial u_{i}}, \\
G_{i j}=\left(2 u_{j}+1\right)\left(\phi\left(-u_{j}-1\right) \bar{\alpha}\left(u_{j}\right) \Lambda_{1}\left(u_{j}\right) Q\left(-u_{j}, \bar{u}_{i j}\right)-\phi\left(u_{j}\right) \bar{\delta}\left(u_{j}\right) \Lambda_{2}\left(u_{j}\right) Q\left(u_{j}+1, \bar{u}_{i j}\right)\right), & \text { for } i \neq j .
\end{aligned}
$$


Evaluating the derivative in $G_{i i}$ and using the Bethe equations, we obatin the following explicit expression,

$$
\begin{aligned}
G_{i i}= & -\phi\left(-u_{i}-1\right) \bar{\alpha}\left(u_{i}\right) \Lambda_{1}\left(u_{i}\right) Q\left(-u_{i}, \bar{u}_{i}\right)\left(\left(2 u_{i}-1\right) \sum_{k \neq i}^{N} \frac{1}{Q\left(-u_{i}, u_{k}\right)}+\left(\frac{1}{u_{i}}-\frac{\partial_{u_{i}} \tilde{\phi}\left(u_{i}\right)}{\tilde{\phi}\left(u_{i}\right)}+\frac{\partial_{u_{i}} \bar{\alpha}\left(u_{i}\right)}{\bar{\alpha}\left(u_{i}\right)}\right)\right) \\
& +\phi\left(u_{i}\right) \bar{\delta}\left(u_{i}\right) \Lambda_{2}\left(u_{i}\right) Q\left(u_{i}+1, \bar{u}_{i}\right)\left(\left(2 u_{i}+3\right) \sum_{k \neq i}^{N} \frac{1}{Q\left(u_{i}+1, u_{k}\right)}+\left(\frac{1}{u_{i}+1}-\frac{\partial_{u_{i}} \tilde{\phi}\left(u_{i}\right)}{\tilde{\phi}\left(u_{i}\right)}+\frac{\partial_{u_{i}} \bar{\delta}\left(u_{i}\right)}{\bar{\delta}\left(u_{i}\right)}\right)\right) \\
& -\frac{\partial \Lambda_{1}\left(u_{i}\right)}{\partial u_{i}}\left(\phi\left(-u_{i}-1\right) \bar{\alpha}\left(u_{i}\right) Q\left(-u_{i}, \bar{u}_{i}\right)-\frac{\rho \tilde{\phi}\left(u_{i}\right) \Lambda_{2}\left(u_{i}\right)}{2 u_{i}+1}\right) \\
& +\frac{\partial \Lambda_{2}\left(u_{i}\right)}{\partial u_{i}}\left(\phi\left(u_{i}\right) \bar{\delta}\left(u_{i}\right) Q\left(u_{i}+1, \bar{u}_{i}\right)+\frac{\rho \tilde{\phi}\left(u_{i}\right) \Lambda_{1}\left(u_{i}\right)}{2 u_{i}+1}\right) .
\end{aligned}
$$

Construction of the conjecture. To obtain the conjecture of the modified Slavnov formula in the previous paragraph, we have used the same procedure from the twisted XXX chain case considered in [7]. The construction of, for instance (42), is based on the following line of reasoning:

I. The modified formula (42) must be reduced to the formula of the case with diagonal boundaries $(\rho=0)$, which is known in the literature [34, 19, 20. In our notation, it reads,

$$
\tilde{S}_{d}^{M}(\bar{u}, \bar{v})=\Lambda_{2}(\bar{v}) \prod_{i=1}^{M} \frac{2 v_{i}+1}{v_{i}+q} \prod_{j<i} \frac{v_{i}+v_{j}+2}{v_{i}+v_{j}} \frac{\operatorname{Det}_{M}\left(\frac{\partial}{\partial v_{i}} \Lambda_{d}^{M}\left(u_{j}, \bar{v}\right)\right)}{\operatorname{Det}_{M}\left(V\left(u_{i}, v_{j}\right)\right)},
$$

where $v_{i}$ for $i=1, \ldots, M$ satisfies $E_{d}^{M}\left(v_{i}, \bar{v}_{i}\right)=0$. From this, we expect that the modified formula contains the Jacobian of the inhomogeneous eigenvalue $\Lambda^{N}(u, \bar{v})$ given by (38) and an additional factor to replace $\Lambda_{2}(\bar{v})$, namely,

$$
\tilde{S}^{N}(\bar{u}, \bar{v})=\zeta^{N} Z^{N}(\bar{u}, \bar{v}) \frac{\operatorname{Det}_{N}\left(\frac{\partial}{\partial v_{i}} \Lambda^{N}\left(u_{j}, \bar{v}\right)\right)}{\operatorname{Det}_{N}\left(V\left(u_{i}, v_{j}\right)\right)}
$$

where the constant $\zeta$ and the function $Z^{N}(\bar{u}, \bar{v})$ are to be fixed.

II. We recall that the factor $\Lambda_{2}(\bar{v})$ in (48) comes from using, in some expression for the off-shell scalar product, the Bethe equations without solving it explicitly, i.e., by writing $\Lambda_{1}\left(v_{i}\right) \sim \Lambda_{2}\left(v_{i}\right)$ from $E_{d}^{M}\left(v_{i}, \bar{v}_{i}\right)=0$ in (31). For the general model, since the Bethe equations (38) are quadratic in $\Lambda_{1,2}\left(v_{i}\right)$, the only way to use the Bethe equations is by expressing the term $\Lambda_{1}\left(v_{i}\right) \Lambda_{2}\left(v_{i}\right)$ through (38) as follows,

$$
\Lambda_{1}\left(v_{i}\right) \Lambda_{2}\left(v_{i}\right)=\frac{\left(2 v_{i}+1\right) Q\left(v_{i}, \bar{v}_{i}\right)}{\rho \tilde{\phi}\left(v_{i}\right)}\left(\phi\left(-v_{i}-1\right) \bar{\alpha}\left(v_{i}\right) \Lambda_{1}\left(v_{i}\right) f\left(v_{i}, \bar{v}_{i}\right)-\phi\left(v_{i}\right) \bar{\delta}\left(v_{i}\right) \Lambda_{2}\left(v_{i}\right) h\left(v_{i}, \bar{v}_{i}\right)\right),
$$

for $i=1, \ldots, N$.

III. The function $Z^{N}(\bar{u}, \bar{v})$ can be fixed from a recursion relation on the scalar product up to the constant $\zeta$.

IV. The constant $\zeta$ can be fixed from the case $N=1$ which we consider explicitly. 
Considering the above points, let us fix the function $Z^{N}(\bar{u}, \bar{v})$ through the action of the $\overline{\mathscr{C}}(u)$ over the Bethe vector (23), namely,

$$
\begin{gathered}
\overline{\mathscr{C}}(u)\left|\Psi^{N}(\bar{v})\right\rangle=-\left(\frac{\rho}{\xi^{-}}\right)^{2} \overline{\mathscr{B}}(u)\left|\Psi^{N}(\bar{v})\right\rangle+\frac{\rho}{\xi^{-}}\left(\phi(-u-1) \Lambda_{1}(u) f(u, \bar{v})-\Lambda_{2}(u) h(u, \bar{v})\right)\left|\Psi^{N}(\bar{v})\right\rangle \\
-\frac{\rho}{\xi^{-}} \sum_{i=1}^{N} w\left(u, v_{i}\right)\left(2 v_{i} \Lambda_{1}\left(v_{i}\right) g\left(u, v_{i}\right) f\left(v_{i}, \bar{v}_{i}\right)\right. \\
\left.+\left(1+2 v_{i}\right) \Lambda_{2}\left(v_{i}\right) k\left(v_{i}, u\right) h\left(v_{i}, \bar{v}_{i}\right)\right)\left|\Psi^{N}\left(\left\{u, \bar{v}_{i}\right\}\right)\right\rangle \\
\quad+\sum_{i=1}^{N} H_{i}(u, \bar{v})\left|\Psi^{N-1}\left(\bar{v}_{i}\right)\right\rangle+\sum_{i<j}^{N} H_{i j}(u, \bar{v})\left|\Psi^{N-1}\left(\left\{u, \bar{v}_{i j}\right\}\right)\right\rangle,
\end{gathered}
$$

where the auxiliary functions $H_{i}(u, \bar{v})$ and $H_{i j}(u, \bar{v})$ are given in appendix $\mathrm{A}$ Taking into account the off-shell action (33), the equation (51) leads to,

$$
S^{N}(\bar{u} \mid \bar{v}) \sim \prod_{i=1}^{N} \frac{\Lambda_{g}\left(u_{i}, \bar{v}\right)}{2\left(u_{i}+1\right)}\left\langle 0 \mid \Psi^{N}(\bar{v})\right\rangle+\cdots
$$

where we identify a leading term with $N$ quadratic terms: $\Lambda_{1}\left(u_{i}\right) \Lambda_{2}\left(u_{i}\right)$ with $i=1, \ldots N$. This term is the only invariant one, of order $3 N$ in the $\Lambda_{1,2}\left(v_{i}\right)$, under the prescription (50). This suggests to us to identify the unknown function in (49) as,

$$
Z^{N}(\bar{u}, \bar{v})=W_{0}^{N}(\bar{v})
$$

Indeed, the leading term of the Jacobian in $\Lambda_{1}\left(v_{i}\right) \Lambda_{2}\left(v_{i}\right)$ is given by,

$$
\operatorname{Det}_{N}\left(\frac{\partial}{\partial v_{i}} \Lambda_{g}^{N}\left(u_{j}, \bar{v}\right)\right)=\prod_{i=1}^{N} \frac{\Lambda_{g}\left(u_{i}, \bar{v}\right)}{2\left(u_{i}+1\right)} \operatorname{Det}_{N}\left(V\left(u_{i}, v_{j}\right)\right)
$$

which makes the asymptotic behavior of (49) consistent with (52). Let us also note that in the diagonal boundaries limit, $\rho=0$, we have

$$
W_{0}^{N}(\bar{v})=\prod_{i=1}^{N} \frac{-2 v_{i}-1}{v_{i}+q} \Lambda_{2}\left(v_{i}\right) \prod_{i<j} \frac{v_{i}+v_{j}+2}{v_{i}+v_{j}},
$$

and the usual formula (48) is recovered, up to a constant. The remaining task is to fix the constant $\zeta$ in (49). In order to do that, we need to find a good parametrization for off-shell scalar product such that the prescription (50) can be used. In the simplest case, $N=1$, it turns out that the off-shell scalar product can be written as,

$$
S^{1}\left(u_{1} \mid v_{1}\right)=\frac{\rho-2}{2(\rho-1)^{2}}\left((\rho-1) S_{d}^{1}\left(u_{1} \mid v_{1}\right)+\left(\frac{\Lambda_{g}^{1}\left(u_{1}, v_{1}\right) W_{0}^{1}\left(v_{1}\right)}{2\left(u_{1}+1\right)}+\frac{\Lambda_{g}^{1}\left(v_{1}, u_{1}\right) W_{0}^{1}\left(u_{1}\right)}{2\left(v_{1}+1\right)}\right)\right)
$$

where

$$
\begin{gathered}
S_{d}^{1}\left(u_{1} \mid v_{1}\right)=\left(s\left(u_{1}, v_{1}\right)+x\left(u_{1}, v_{1}\right)\right) \Lambda_{1}\left(u_{1}\right) \Lambda_{1}\left(v_{1}\right)+y\left(u_{1}, v_{1}\right) \Lambda_{2}\left(u_{1}\right) \Lambda_{1}\left(v_{1}\right)+r\left(u_{1}, v_{1}\right) \Lambda_{1}\left(u_{1}\right) \Lambda_{2}\left(v_{1}\right) \\
+q\left(u_{1}, v_{1}\right) \Lambda_{1}\left(v_{1}\right) \Lambda_{2}\left(u_{1}\right)+w\left(u_{1}, v_{1}\right) \Lambda_{2}\left(u_{1}\right) \Lambda_{2}\left(v_{1}\right),
\end{gathered}
$$

is the diagonal contribution to the scalar product. In the form (56), the use of (50) leads us directly to,

$$
\tilde{S}^{1}\left(u_{1} \mid v_{1}\right)=\frac{\rho-2}{2(\rho-1)^{2}} W_{0}^{1}\left(v_{1}\right) \frac{\frac{\partial}{\partial v_{1}} \Lambda^{1}\left(u_{1}, v_{1}\right)}{V\left(u_{1}, v_{1}\right)},
$$


fixing thus the desired constant. To find a convenient off-shell representation for the scalar product for general $N$ that allows us to use (49) remains an open problem. For example, the off-shell scalar product obtained from the projection of the Bethe vector on the diagonal operator basis, see appendix B gives us,

$$
S^{1}\left(u_{1} \mid v_{1}\right)=\left(\frac{\rho-2}{2(\rho-1)}\right)^{2} S_{d}^{1}\left(u_{1} \mid v_{1}\right)+\frac{\rho(\rho-2)}{(2(\rho-1))^{2}} W_{0}^{1}\left(u_{1}\right) W_{0}^{1}\left(v_{1}\right)
$$

Here the use of the prescription (50) for the quadratic term cannot be applied. Alternatively, from the use of the action (51), we obtain,

$$
\begin{aligned}
& S^{1}\left(u_{1} \mid v_{1}\right)=S_{d}^{1}\left(u_{1} \mid v_{1}\right)-\frac{\rho}{2(\rho-1)^{2}}\left(\frac{\Lambda_{g}^{1}\left(u_{1}, v_{1}\right) W_{0}^{1}\left(v_{1}\right)}{2\left(u_{1}+1\right)}+\frac{\Lambda_{g}^{1}\left(v_{1}, u_{1}\right) W_{0}^{1}\left(u_{1}\right)}{2\left(v_{1}+1\right)}\right) \\
& +\frac{\rho}{2(\rho-1)}\left(\phi\left(-v_{1}-1\right) \Lambda_{1}\left(v_{1}\right)\left(\frac{u_{1}+v_{1}-1}{u_{1}+v_{1}+1} \phi\left(-u_{1}-1\right) \Lambda_{1}\left(u_{1}\right)-\frac{u_{1}-v_{1}+2}{u_{1}-v_{1}} \Lambda_{2}\left(u_{1}\right)\right)\right. \\
& \left.-\Lambda_{2}\left(v_{1}\right)\left(\frac{u_{1}-v_{1}-2}{u_{1}-v_{1}} \phi\left(-u_{1}-1\right) \Lambda_{1}\left(u_{1}\right)-\frac{u_{1}+v_{1}+3}{u_{1}+v_{1}+1} \Lambda_{2}\left(u_{1}\right)\right)\right),
\end{aligned}
$$

where we observe the presence of quadratic terms that can be simplified by (50). However, it does not lead directly to the modified Slavnov formula due to the presence of additional contributions. We remark that all the representations (56/59]60) are the same, as expected, when we use the explicit form of $\Lambda_{1,2}(u)$.

By any means, the $N=1$ case is already enough to obtain the constant and thus to propose the general formulas. We have numerically verified that both (40/42) and (46) are valid for $N=2,3$.

Discussion. We have obtained, in the framework of the modified algebraic Bethe ansatz, a compact expression for the scalar product between an on-shell Bethe vector and its off-shell dual for the isotropic spin-1/2 Heisenberg chain on the segment with general integrable boundaries. It is a modified Slavnov formula, in the sense that new factors appear, when compared with the diagonal boundary or periodic cases. Many interesting questions remain to be investigated. The first one is to obtain a proof of our conjecture. Next, we should consider the evaluation of form factors and correlations functions of the model. The XXZ case can be also considered, and the result has the same form of (40[42), with $\Lambda^{N}(u, \bar{u})$ and $\operatorname{Det}_{N}(V(u, v))$ replaced by the corresponding $q$-deformed versions. The factor $W_{0}^{N}(\bar{u})$ will be given by (78) with $W_{i-1}^{i}\left(u_{1} \mid u_{2}, \ldots, u_{i}\right)=\Lambda_{p s}^{i-1}\left(u_{1},\left\{u_{2}, \ldots, u_{i}\right\}\right)$, using the notaion of [6]. It remains to find the overall constant and to prove the conjecture.

Acknowledgements. We would like to thank R. Nepomechie for discussions. R.A.P would like to thanks the hospitality of the Laboratoire de Physique Théorique et Modélisation at the Université de Cergy-Pontoise where a part of this work was done. S.B. is supported by the Université de Cergy-Pontoise post doctoral fellowship and by a public grant as part of the Investissement d'avenir project, reference ANR-11-LABX0056-LMH, LabEx LMH. R.A.P. is supported by Sao Paulo Research Foundation (FAPESP), grants \# 2014/00453-8 and \# 2014/20364-0.

\section{Appendix A. Commutation Relations and functions}

Using reflection equation (4), we can obtain the exchange relations between the operators $\{\mathscr{A}, \mathscr{B}, \mathscr{C}, \mathscr{D}\}$. A list of relevant ones for this work is,

$$
\mathscr{B}(u) \mathscr{B}(v)=\mathscr{B}(v) \mathscr{B}(u),
$$




$$
\begin{aligned}
\mathscr{C}(u) \mathscr{C}(v)= & \mathscr{C}(v) \mathscr{C}(u), \\
\mathscr{A}(u) \mathscr{B}(v)= & f(u, v) \mathscr{B}(v) \mathscr{A}(u)+g(u, v) \mathscr{B}(u) \mathscr{A}(v)+w(u, v) \mathscr{B}(u) \mathscr{D}(v), \\
\mathscr{C}(v) \mathscr{A}(u)= & f(u, v) \mathscr{A}(u) \mathscr{C}(v)+g(u, v) \mathscr{A}(v) \mathscr{C}(u)+w(u, v) \mathscr{D}(v) \mathscr{C}(u), \\
\mathscr{D}(u) \mathscr{B}(v)= & h(u, v) \mathscr{B}(v) \mathscr{D}(u)+k(u, v) \mathscr{B}(u) \mathscr{D}(v)+n(u, v) \mathscr{B}(u) \mathscr{A}(v), \\
\mathscr{C}(v) \mathscr{D}(u)= & h(u, v) \mathscr{D}(u) \mathscr{C}(v)+k(u, v) \mathscr{D}(v) \mathscr{C}(u)+n(u, v) \mathscr{A}(v) \mathscr{C}(u), \\
\mathscr{C}(u) \mathscr{B}(v)= & \mathscr{B}(v) \mathscr{C}(u)+s(u, v) \mathscr{A}(u) \mathscr{A}(v)+x(u, v) \mathscr{A}(v) \mathscr{A}(u)+y(u, v) \mathscr{D}(u) \mathscr{A}(v) \\
& +r(u, v) \mathscr{A}(u) \mathscr{D}(v)+q(u, v) \mathscr{A}(v) \mathscr{D}(u)+w(u, v) \mathscr{D}(u) \mathscr{D}(v),
\end{aligned}
$$

where

$$
\begin{array}{rlrl}
f(u, v) & =\frac{(u-v-1)(u+v)}{(u-v)(u+v+1)}, \quad g(u, v)=\frac{2 v}{(2 v+1)(u-v)}, & w(u, v)=\frac{-1}{(u+v+1)}, \\
(68) h(u, v) & =\frac{(u-v+1)(u+v+2)}{(u-v)(u+v+1)}, & k(u, v)=\frac{-2(u+1)}{(u-v)(2 u+1)}, & n(u, v)=\frac{4 v(u+1)}{(u+v+1)(2 v+1)(2 u+1)},
\end{array}
$$

and

$$
\begin{aligned}
& x(u, v)=\frac{2 u(u-v+1)}{(2 u+1)(u+v+1)(u-v)}, \quad s(u, v)=-\frac{2 u}{(2 u+1)(2 v+1)(u-v)}, \\
& q(u, v)=\frac{(u+v)}{(u+v+1)(u-v)}, \quad r(u, v)=-\frac{2 u}{(2 u+1)(u-v)}, \quad y(u, v)=-\frac{1}{(u+v+1)(2 v+1)} .
\end{aligned}
$$

Along the main text we use frequently the functions,

$$
\phi(u)=\frac{2(u+1)}{2 u+1}, \quad F(u, v)=-\frac{\phi(u)(2 u+1)}{\phi(v) Q(u, v)}, \quad Q(u, v)=(u-v)(u+v+1) .
$$

In addition, we have the following auxiliary functions entering equation (51),

$$
\begin{gathered}
H_{k}(u, \bar{u})=\Lambda_{1}(u)\left(\Lambda_{1}\left(u_{k}\right)\left(s\left(u, u_{k}\right)+x\left(u, u_{k}\right)\right) f\left(u, \bar{u}_{k}\right) f\left(u_{k}, \bar{u}_{k}\right)+\Lambda_{2}\left(u_{k}\right) r\left(u, u_{k}\right) f\left(u, \bar{u}_{k}\right) h\left(u_{k}, \bar{u}_{k}\right)\right) \\
+\Lambda_{2}(u)\left(\Lambda_{1}\left(u_{k}\right)\left(q\left(u, u_{k}\right)+y\left(u, u_{k}\right)\right) h\left(u, \bar{u}_{k}\right) f\left(u_{k}, \bar{u}_{k}\right)+\Lambda_{2}\left(u_{k}\right) w\left(u, u_{k}\right) h\left(u, \bar{u}_{k}\right) h\left(u_{k}, \bar{u}_{k}\right)\right) \\
H_{k l}(u, \bar{u})=\Lambda_{1}\left(u_{k}\right)\left(\Lambda_{1}\left(u_{l}\right) \alpha_{11}\left(u, u_{k}, u_{l}\right) f\left(u_{k}, \bar{u}_{k l}\right) f\left(u_{l}, \bar{u}_{k l}\right)+\Lambda_{2}\left(u_{l}\right) \alpha_{12}\left(u, u_{k}, u_{l}\right) f\left(u_{k}, \bar{u}_{k l}\right) h\left(u_{l}, \bar{u}_{k l}\right)\right) \\
+\Lambda_{2}\left(u_{k}\right)\left(\Lambda_{1}\left(u_{l}\right) \alpha_{21}\left(u, u_{k}, u_{l}\right) h\left(u_{k}, \bar{u}_{k l}\right) f\left(u_{l}, \bar{u}_{k l}\right)+\Lambda_{2}\left(u_{l}\right) \alpha_{22}\left(u, u_{l}, u_{k}\right) h\left(u_{k}, \bar{u}_{k l}\right) h\left(u_{l}, \bar{u}_{k l}\right)\right)
\end{gathered}
$$

with

$$
\begin{aligned}
& \alpha_{11}\left(u, u_{k}, u_{l}\right) \\
& =g\left(u, u_{l}\right)\left(s\left(u, u_{k}\right) f\left(u_{k}, u_{l}\right)+f\left(u_{k}, u\right) x\left(u, u_{k}\right)\right)+n\left(u, u_{l}\right)\left(y\left(u, u_{k}\right) f\left(u_{k}, u_{l}\right)+f\left(u_{k}, u\right) q\left(u, u_{k}\right)\right) \\
& +g\left(u, u_{k}\right)\left(s\left(u, u_{k}\right) g\left(u_{k}, u_{l}\right)+r\left(u, u_{k}\right) n\left(u_{k}, u_{l}\right)\right)+n\left(u, u_{k}\right)\left(y\left(u, u_{k}\right) g\left(u_{k}, u_{l}\right)+w\left(u, u_{k}\right) n\left(u_{k}, u_{l}\right)\right) \text {, } \\
& \alpha_{12}\left(u, u_{k}, u_{l}\right) \\
& =k\left(u, u_{l}\right)\left(f\left(u_{k}, u\right) q\left(u, u_{k}\right)+f\left(u_{k}, u_{l}\right) y\left(u, u_{k}\right)\right)+w\left(u, u_{l}\right)\left(f\left(u_{k}, u_{l}\right) s\left(u, u_{k}\right)+f\left(u_{k}, u\right) x\left(u, u_{k}\right)\right) \\
& +g\left(u, u_{k}\right)\left(k\left(u_{k}, u_{l}\right) r\left(u, u_{k}\right)+s\left(u, u_{k}\right) w\left(u_{k}, u_{l}\right)\right)+n\left(u, u_{k}\right)\left(k\left(u_{k}, u_{l}\right) w\left(u, u_{k}\right)+y\left(u, u_{k}\right) w\left(u_{k}, u_{l}\right)\right) \text {, } \\
& \alpha_{21}\left(u, u_{k}, u_{l}\right) \\
& =r\left(u, u_{k}\right)\left(g\left(u, u_{l}\right) h\left(u_{k}, u_{l}\right)+n\left(u_{k}, u_{l}\right) w\left(u, u_{k}\right)\right)+g\left(u_{k}, u_{l}\right)\left(k\left(u, u_{k}\right) y\left(u, u_{k}\right)+s\left(u, u_{k}\right) w\left(u, u_{k}\right)\right) \\
& +w\left(u, u_{k}\right)\left(h\left(u_{k}, u_{l}\right) n\left(u, u_{l}\right)+k\left(u, u_{k}\right) n\left(u_{k}, u_{l}\right)\right) \text {, }
\end{aligned}
$$




$$
\begin{aligned}
& \alpha_{22}\left(u, u_{k}, u_{l}\right) \\
& \quad=r\left(u, u_{k}\right)\left(h\left(u_{k}, u_{l}\right) w\left(u, u_{l}\right)+k\left(u_{k}, u_{l}\right) w\left(u, u_{k}\right)\right)+w\left(u, u_{k}\right)\left(h\left(u_{k}, u_{l}\right) k\left(u, u_{l}\right)+k\left(u, u_{k}\right) k\left(u_{k}, u_{l}\right)\right) \\
& \quad+w\left(u_{k}, u_{l}\right)\left(k\left(u, u_{k}\right) y\left(u, u_{k}\right)+s\left(u, u_{k}\right) w\left(u, u_{k}\right)\right) .
\end{aligned}
$$

\section{Appendix B. Projection of the Bethe vector}

Using the commutation relations given in appendix $\mathrm{A}$ and the representation theory (14), the Bethe vector (23) can be expanded in terms of the operator $\mathscr{B}(u)$ in the following way,

$$
\overline{\mathscr{B}}(\bar{u})|0\rangle=\left(\frac{(\rho-2) \xi^{-}}{2(\rho-1) \xi^{+}}\right)^{N} \sum_{i=0}^{N} \sum_{\bar{u} \rightarrow\left\{\bar{u}_{\mathrm{I}}, \bar{u}_{\mathrm{II}}\right\}}\left(\frac{\rho}{\xi^{-}}\right)^{N-i} W_{i}^{N}\left(\bar{u}_{\mathrm{I}} \mid \bar{u}_{\mathrm{II}}\right) \mathscr{B}\left(\bar{u}_{\mathrm{II}}\right)|0\rangle
$$

with $\# \bar{u}_{\mathrm{I}}=i, \# \bar{u}_{\mathrm{I}}=N-i$ a partition of the set $\bar{u}$. The sum is over all ordered partitions, denoted $\bar{u} \rightarrow\left\{\bar{u}_{\mathrm{I}}, \bar{u}_{\mathrm{II}}\right\}$. The coefficient is given by

$$
W_{i}^{N}\left(u_{1}, \ldots, u_{N-i} \mid u_{N-i+1}, \ldots, u_{N}\right)=\operatorname{Sym}_{u_{1}, \ldots, u_{N-i}}^{N-i}\left(\prod_{j=1}^{N-i} W_{N-j}^{N+1-j}\left(u_{j} \mid u_{j+1}, \ldots, u_{N}\right)\right)
$$

where

$$
W_{i-1}^{i}\left(u_{1} \mid u_{2}, \ldots, u_{i}\right)=\phi\left(-u_{1}-1\right) \Lambda_{1}\left(u_{1}\right) f\left(u_{1}, \bar{u}_{1}\right)-\Lambda_{2}\left(u_{1}\right) h\left(u_{1}, \bar{u}_{1}\right),
$$

and

$$
\operatorname{Sym}_{\bar{u}}^{M}(F(\bar{u}))=\frac{1}{M !} \sum_{\sigma \in S_{M}} F\left(\bar{u}^{\sigma}\right)
$$

with $\bar{u}^{\sigma}=\left\{u_{\sigma(1)}, \ldots, u_{\sigma(M)}\right\}$ an element of the permutation group $S_{M}$. In the same way, the dual Bethe vector (24) in terms of the operator $\mathscr{C}(u)$ is given by,

$$
\langle 0| \overline{\mathscr{C}}(\bar{u})=\left(\frac{(\rho-2) \xi^{+}}{2(\rho-1) \xi^{-}}\right)^{N} \sum_{i=0}^{N} \sum_{\bar{u} \rightarrow\left\{\bar{u}_{\mathrm{I}}, \bar{u}_{\mathrm{II}}\right\}}\left(\frac{\rho}{\xi^{+}}\right)^{N-i} W_{i}^{N}\left(\bar{u}_{\mathrm{I}} \mid \bar{u}_{\mathrm{II}}\right)\langle 0| \mathscr{C}\left(\bar{u}_{\mathrm{II}}\right) .
$$

\section{REFERENCES}

[1] Cirilo António N. , Manojlović N. and Salom I., Algebraic Bethe ansatz for the XXX chain with triangular boundaries and Gaudin model, Nucl. Phys. B 889 (2014) 87, arXiv:1405.7398

[2] Avan J., Belliard S., Grosjean N. and Pimenta R.A., Modified algebraic Bethe ansatz for XXZ chain on the segment - III - Proof, , Nucl. Phys. B 899 (2015) 229, arXiv:1506.02147.

[3] Belliard S. and Crampé N., Heisenberg XXX Model with General Boundaries: Eigenvectors from Algebraic Bethe Ansatz, SIGMA 9 (2013), 072, arXiv:1309.6165.

[4] Belliard S., Crampé N. and Ragoucy E., Algebraic Bethe ansatz for open XXX model with triangular boundary matrices, Lett. Math. Phys. 103 (2013) 493, arXiv:1209.4269

[5] Belliard S. , Modified algebraic Bethe ansatz for XXZ chain on the segment - I: Triangular cases, Nucl. Phys. B 892 (2015) 1, arXiv: 1408.4840.

[6] Belliard S. and Pimenta R.A., Modified algebraic Bethe ansatz for XXZ chain on the segment - II - general cases, Nucl. Phys. B 894 (2015) 527, arXiv:1412.7511

[7] Belliard S. and Pimenta R.A., Slavnov and Gaudin-Korepin formulas for models without U(1) symmetry: the twisted XXX chain, SIGMA 11 (2015) 099, arXiv:1506.06550.

[8] Baseilhac P. and Koizumi K. , Exact spectrum of the XXZ open spin chain from the q-Onsager algebra representation theory, J. Stat. Mech. (2007) P09006, arXiv:hep-th/0703106

[9] Cao J., Lin H.-Q. , Shi K. and Wang Y., Exact solutions and elementary excitations in the XXZ spin chain with unparallel boundary fields, Nucl. Phys. B663 (2003) 487, arXiv: cond-mat/0212163

[10] Cao J., Yang W., Shi K., Wang Y., Off-diagonal Bethe ansatz solution of the XXX spin-chain with arbitrary boundary conditions, Nucl. Phys. B $\mathbf{8 7 5}$ (2013) 152, arXiv:1306.1742

[11] Crampé N., Algebraic Bethe ansatz for the totally asymmetric simple exclusion process with boundaries, J. Phys. A: Math. Theor. 48 (2015) 08FT01, arXiv: 1411.7954 
[12] de Vega H. J. and Gonzalez-Ruiz A., Boundary K-matrices for the six vertex and the n(2n-1) $A_{n-1}$ vertex models, J. Phys. A26 (1993) 519, arXiv:hep-th/9211114

[13] Faldella S., Kitanine N. and Niccoli G., Complete spectrum and scalar products for open spin-1/2 XXZ quantum chains with non-diagonal boundary terms, J. Stat. Mech. (2014) P01011, arXiv:1307.3960

[14] Frahm H., Grelik J.H., Seel A. and Wirth T., Functional Bethe ansatz methods for the open XXX chain, J. Phys. A44 (2011) 015001 and arXiv:1009.1081.

[15] Frahm H., Seel A. and Wirth T., Separation of Variables in the open XXX chain, Nucl. Phys. B802 (2008) 351 and arXiv:0803.1776.

[16] Galleas W., Functional relations from the Yang-Baxter algebra: Eigenvalues of the XXZ model with non-diagonal twisted and open boundary conditions, Nucl. Phys. B790 (2008) 524, arXiv:0708.0009

[17] Gaudin M., La fonction d'onde de Bethe, Masson, Paris, 1983

[18] Gaudin M., McCoy B. and Wu T.T. Normalization sum for the Bethe s hypothesis wave functions of the Heisenberg-Ising chain Phys. Rev. D23 (1981) 417

[19] Kitanine N., Kozlowski K.K., Maillet J.M., Niccoli G., Slavnov N.A. and Terras V., Correlation functions of the open XXZ chain I, JSTAT 0710 (2007) P10009, arXiv:0707.1995.

[20] Kitanine N., Kozlowski K.K., Maillet J.M., Niccoli G., Slavnov N.A. and Terras V., Correlation functions of the open XXZ chain II, JSTAT 07 (2008) P07010, arXiv:0803.3305

[21] Kitanine N., Maillet J.M., Niccoli G. and Terras V., On determinant representations of scalar products and form factors in the SoV approach: the XXX case, J. Phys. A: Math. Theor. 49 (2016) 104002, arXiv:1506.02630

[22] Korepin V.E., Calculations of norms of Bethe wave functions, Commun. Math. Phys. 86 (1982), 391

[23] Korepin V.E., Bogoliubov N.M. and Izergin A.G., Quantum Inverse Scattering Method and Correlation Functions, Cambridge Univ. Press (1993).

[24] Kitanine N., Maillet J.M. and Niccoli G., Open spin chains with generic integrable boundaries: Baxter equation and Bethe ansatz completeness from separation of variables, J. Stat. Mech. (2014) P05015, arXiv:1401.4901

[25] Lazarescu A., Pasquier V., Bethe Ansatz and Q-operator for the open ASEP J. Phys. A: Math. Theor. 47 (2014) 295202, arXiv:1403.6963

[26] Nepomechie R.I., Bethe Ansatz solution of the open XXZ chain with nondiagonal boundary terms, J. Phys. A37 (2004) 433, arXiv:hep-th/0304092.

[27] Nepomechie R.I., "An inhomogeneous T-Q equation for the open XXX chain with general boundary terms: completeness and arbitrary spin," J. Phys. A: Math. Theor. 46 (2013) 442002

[28] Niccoli G., Non-diagonal open spin-1/2 XXZ quantum chains by separation of variables: Complete spectrum and matrix elements of some quasi-local operators, J. Stat. Mech. (2012) P10025 and arXiv:1206.0646.

[29] Pimenta R.A. and Lima-Santos A., Algebraic Bethe ansatz for the six vertex model with upper triangular $K$-matrices, J. Phys. A: Math. Theor. 46 (2013) 455002, arXiv:1308.4446

[30] Sklyanin E.K., Boundary conditions for integrable quantum systems, J. Phys. A21 (1988) 2375.

[31] Sklyanin E.K., Takhtadzhyan L.A. and Faddeev L.D., The Quantum Inverse Problem Method. I, Theor. Math. Phys. 40 (1979) 688.

[32] Slavnov N.A., Calculation of scalar products of wave functions and form factors in the framework of the alcebraic Bethe ansatz, Theor. Math. Phys. 79 (1989) 502.

[33] Wang Y., Yang W.-L., Cao J. and Shi K., Off-Diagonal Bethe Ansatz for Exactly Solvable Models, Springer-Verlag Berlin Heidelberg, (2015).

[34] Wang Y.-S., it The scalar products and the norm of Bethe eigenstates for the boundary XXX Heisenberg spin- $1 / 2$ finite chain, Nucl. Phys. B 622 (2002) 663

[35] Yang W.-L., Chen X., Feng J., Hao K., Hou B.-Y., Shi K.-J., Zhang Y.-Z., Determinant representations of scalar products for the open XXZ chain with non-diagonal boundary terms, JHEP 1101 (2011) 006, arXiv:1011.4719

[36] Zhang X., Li Y.Y., Cao J., Yang W.-L., Shi K., Wang Y., Retrieve the Bethe states of quantum integrable models solved via off-diagonal Bethe ansatz, J. Stat. Mech. (2015) P05014, arXiv:1407.5294v3.

[37] Zhang X., Li Y.Y., Cao J., Yang W.-L., Shi K., Wang Y., Bethe states of the XXZ spin-1/2 chain with arbitrary boundary fields, Nucl. Phys. B893 (2015) 70, arXiv:1412.6905.

Laboratoire de Physique Théorique et Modélisation (CNRS UMR 8089), Université de Cergy-Pontoise, F-95302 Cergy-Pontoise, France

Institut de Physique Théorique, DSM, CEA, URA2306 CNRS Saclay, F-91191 Gif-Sur-Yvette, France

E-mail address: samuel.belliard@u-cergy.fr

Departamento de Física, Universidade Federal de São Carlos, Caixa Postal 676, CeP 13565-905, São Carlos, BRASIL 
SLAVNOV AND GAUDIN-KOREPIN FORMULAS FOR MODELS WITHOUT U(1) SYMMETRY: THE XXX CHAIN ON THE SEGMENIB

Physics Department, University of Miami, P.O. Box 248046, FL 33124, Coral Gables, Usa

E-mail address: pimenta@df.ufscar.br 